\title{
Ceguera legal unilateral y liposucción. Revisión de la literatura y reporte del primer caso en México, octavo en el mundo
}

\section{Liposuction and unilateral legal blindness. Literature review and first case report in Mexico, eight in the world}

Raúl A. VALLARTA RODRÍGUEZ *, Mauricio VALLARTA MACÍAS**

Carlos GONZÁLEZ ALVARADO***, Santiago VALLARTA COMPEÁN****

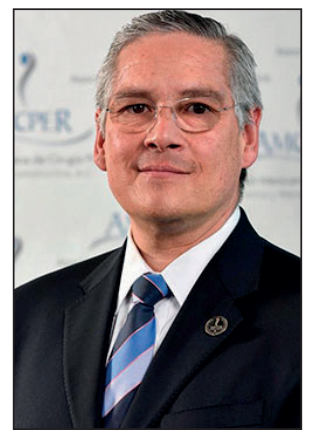

Vallarta Rodríguez R.A.
Resumen

Presentamos el caso de una paciente de 32 años que desarrolló ceguera legal posterior a liposucción y mamoplastia de aumento, evolucionando con anemia transitoria y disminución progresiva de la agudeza visual del ojo izquierdo (20/200). A pesar de la corrección de la anemia y la mejoría del estado general, continuó durante 2 meses con sintomatología ocular.

Se estableció diagnóstico de neuropatía óptica isquémica anterior no arterítica (NOIA) de acuerdo a los hallazgos oftalmológicos.

Revisamos las publicaciones previas para neuritis óptica isquémica anterior después de liposucción y las recomendaciones para prevenir dicha patología.
Abstract

We present the case of a 32-year-old woman who developed legal blindness posterior to liposuction and augmentation mammoplasty, with postoperative anemia and decreased vision of the left eye (20/200). Despite the correction of the anemia and improvement of the general status, the patient continued with vision loss for 2 months.

According to ophthalmologic studies we could establish the diagnosis of non-arteritic ischemic optic neuropathy (NAION).

We review the previous reports in the literature for anterior ischemic optic neuritis after liposuction and the recommendations to prevent this complication.
Palahbras clave Neuropatía óptica, Neuropatía isquémica, Liposucción, Ceguera legal

Nivel de evidencia científica

Recibido [esta versión]

Aceptado 4d Terapéutico

10 febrero/2019

6 noviembre/2019

\section{Key words Optic neuropathy, Ischemic neuropathy,} Liposuction, Legal blindness

Level of evidence
Received [this version]
Accepted

4d Therapeutic

10 February/2019 6 November/2019

Conflicto de intereses: Los autores declaran no tener ningún interés financiero relacionado con el contenido de este artículo. Financiación: No hubo fuentes externas de financiación para este trabajo.

* Cirujano Plástico, Estético y Reconstructivo, Academia Mexicana de Cirugía y Fundación Clínica Médica Sur, Ciudad de México, México.

** Cirujano Oftalmólogo, Centro Atención Visual "CAVAMA", Tepic, Nayarit, México.

*** Médico Residente de Cirugía Plástica, Hospital Central Sur de Alta Especialidad de Petróleos Mexicanos, Ciudad de México, México.

${ }^{* * \star \star}$ Estudiante de Medicina, Universidad Nacional Autónoma de México, Ciudad de México, México. 


\section{Introducción}

En la actualidad, la liposucción es uno de los 5 procedimientos estéticos más comunes en todo el mundo; de acuerdo a la Asociación Americana de Cirugía Plástica Estética (ASAPS), en 2016 se realizaron 414.335 liposucciones solamente en los Estados Unidos. ${ }^{(1)}$

La liposucción es una técnica quirúrgica que se utiliza en Cirugía Plástica para remodelar el contorno corporal extrayendo el tejido adiposo mediante una cánula conectada a una jeringa o a una máquina de aspiración. Fue descrita por primera vez por el ginecólogo italiano Giorgio Fischer en 1974 y en 1977, el francés Yves-Gerard Illouz fue el primero en utilizarla con fines estéticos, adoptando movimientos de vaivén para desprender y aspirar el tejido graso. ${ }^{(2)}$ Desde entonces, el procedimiento ha ido evolucionando a través de mejoras en la tecnología para aplicarla y emplea técnicas más depuradas; así se ha convertido en uno de los procedimientos más habituales en Cirugía Plástica y con ello, los especialistas hemos aprendido algunas variables de morbi-mortalidad que desconocíamos. Al igual que con otros procedimientos quirúrgicos, se han reportado complicaciones sistémicas con la liposucción que van desde el tromboembolismo pulmonar, la intoxicación por lidocaína, hasta los micro y macro embolismos grasos, entre otras.

El término ceguera legal hace referencia a la presencia de una visión sumamente disminuida, de hasta 20/200, y se habla de ceguera total cuando no existe percepción a la luz; ambas son complicaciones poco recogidas en la literatura mundial después de una cirugía no ocular y su asociación con liposucción es prácticamente desconoci$\mathrm{da}$, encontrando solamente reportados a nivel mundial 7 pacientes con ceguera legal o total y liposucción. ${ }^{(3)}$

En este articulo presentamos el primer caso en México y octavo en el mundo de ceguera legal posterior a liposucción, estableciendo el diagnóstico de neuritis óptica isquémica anterior (NOIA) de origen no arterítico. Al mismo tiempo, llevamos a cabo una actualización de las publicaciones existentes en la literatura para llegar a establecer unas conclusiones de prevención y manejo de esta patología.

\section{Caso clínico}

Mujer de 32 años de edad sin antecedentes de importancia. Acude buscando mejoría de su contorno corporal y optamos por realizar liposucción de abdomen y espalda en conjunto con colocación de implantes mamarios. Los exámenes preoperatorios estuvieron dentro de los parámetros normales, destacando una hemoglobina de $14 \mathrm{~g} / \mathrm{dl}$ y un hematocrito del $42 \%$. Se realizó además valoración cardiovascular preoperatoria, sin presentar riesgos cardiológicos que contraindicaran el procedimiento quirúrgico.

Llevamos a cabo el procedimiento bajo anestesia general con técnica tumescente, aplicando solución fisiológica (3litros) y epinefrina (1 ampolla x litro) para obtener un lipoaspirado de $3000 \mathrm{cc}$ de espalda, flancos y abdomen; además colocamos drenaje cerrado en espalda para evitar la formación de seroma. Continuamos con la cirugía mamaria colocando implantes redondos texturizados de $280 \mathrm{cc}$ de volumen en posición retromuscular; dejamos también drenaje cerrado en ambas mamas.

La paciente evolucionó favorablemente después de 4 horas de cirugía y permaneció hospitalizada durante 48 horas. En el primer día de postoperatorio se hizo fórmula roja de control encontrando cifras favorables, con hemoglobina de $11 \mathrm{~g} / \mathrm{dl}$ y hematocrito del $37 \%$. Fue dada de alta hospitalaria con buenas condiciones clínicas.

Acudió posteriormente a consulta externa al cuarto día de postoperatorio con signos vitales estables, refiriendo dolor en áreas quirúrgicas, sin datos de alarma y retiramos los drenajes de ambas mamas dejando el drenaje de la espalda por alto gasto.

Al quinto día de postoperatorio acudió al Servicio de Urgencias de nuestro hospital presentando diaforesis, angustia, palpitaciones, taquicardia y disminución de la visión del ojo izquierdo. Se tomaron exámenes de control encontrando una hemoglobina de $8.5 \mathrm{~g} / \mathrm{dl}$ y un hematocrito del $32 \%$, línea blanca con leucocitosis 14.6 a expensas de neutrofilia. Se realizó tomografía computarizada pulmonar encontrando un mínimo derrame pleural bilateral y se excluyó cualquier otra anormalidad. Se solicitó interconsulta a Oftalmología, destacando los siguientes hallazgos: disminución de la agudeza visual de 20/20 en el ojo derecho y de 20/200 en el ojo izquierdo, examen de fondo de ojo con presencia de pérdida de bordes del nervio óptico, hiperemia difusa y hemorragias peripapilares de predominio en el sector inferior (Fig. 1 y 2). Posteriormente se solicitaron estudios paraclínicos incluyendo campo visual computarizado, 24-2, blanco-blanco, que mostró un defecto altitudinal superior y con lo anterior se estableció el diagnóstico de neuropatía óptica isquémica anterior no arterítica del ojo izquierdo (Fig. 3).

El tratamiento incluyó la transfusión de 2 paquetes globulares que llevaron las cifras de hemoglobina a $10.8 \mathrm{~g} / 1$ y el hematocrito a un $37 \%$ y se estableció tratamiento oftalmológico con prednisona vía oral durante 7 semanas, iniciando con $50 \mathrm{mg}$ la primer semana, $20 \mathrm{mg}$ las siguientes 4 semanas y $10 \mathrm{mg}$ durante las últimas 2 semanas, llevando control oftalmológico con exámenes de fondo de ojo y campo visual. 


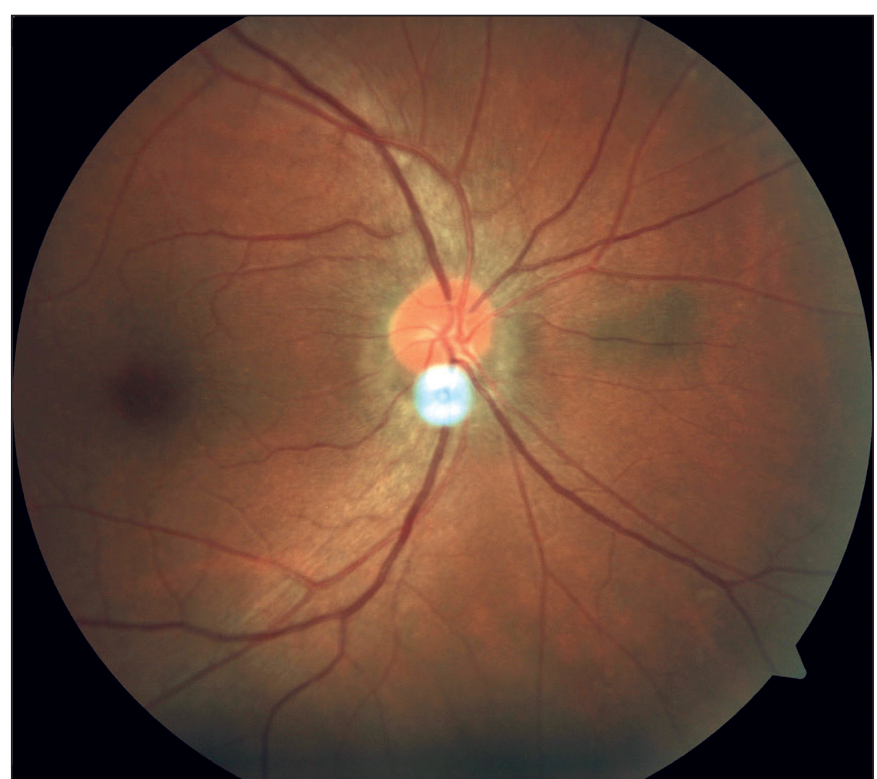

Figura 1. Ojo derecho: fondo de ojo sano, se observa nervio óptico de coIoración amarillo-naranja, bordes bien delimitados, presencia de una excavación fisiológica, emergencia de los vasos y calibre dentro de lo normal.

La paciente evolucionó favorablemente de la cirugía realizada y siguió con tratamiento oftalmológico durante 2 meses hasta lograr la recuperación paulatina y completa de su visión.

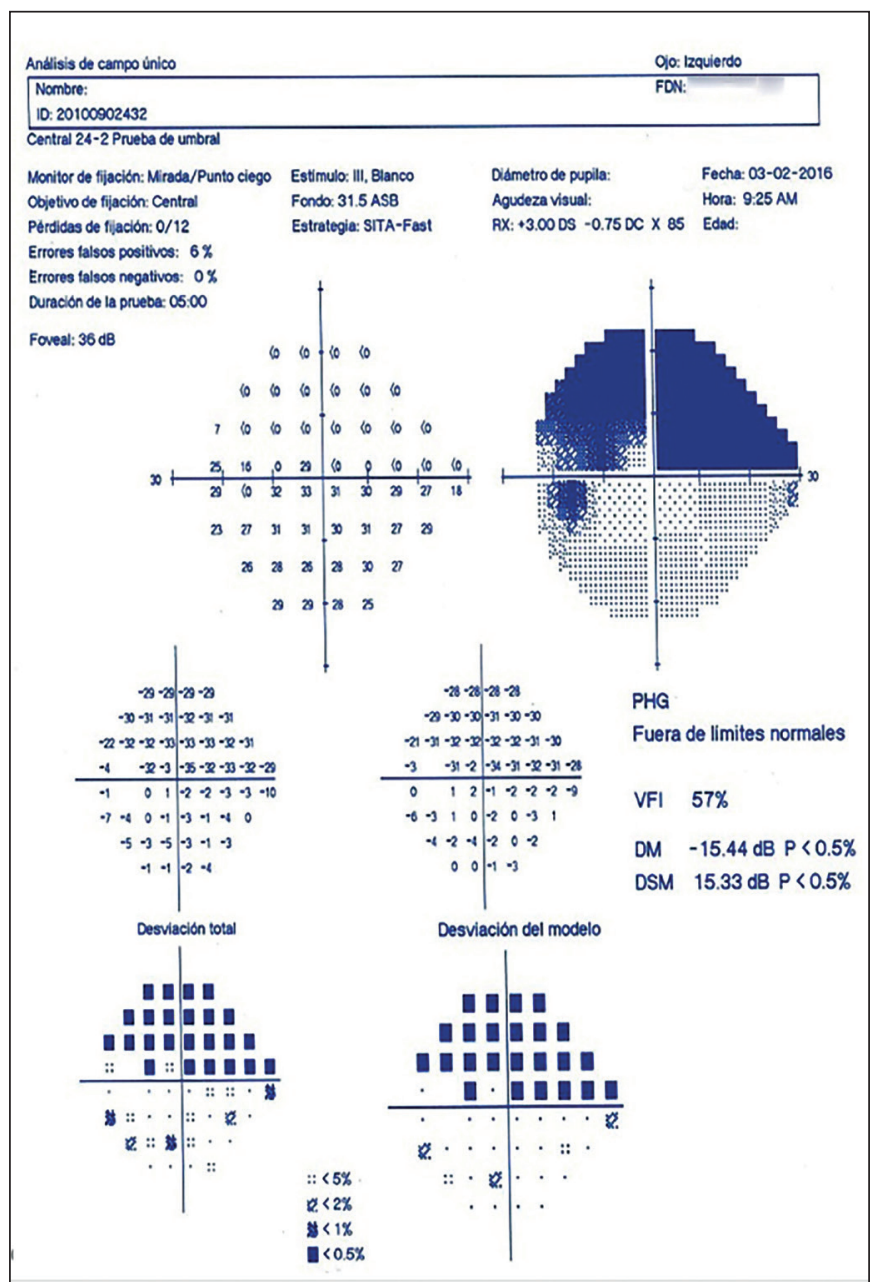

Figura 3. Campo visual computarizado, 24-2, blanco-blanco; el estudio refleja un defecto altitudinal superior (defecto típico en una neuropatía óptica isquémica anterior)

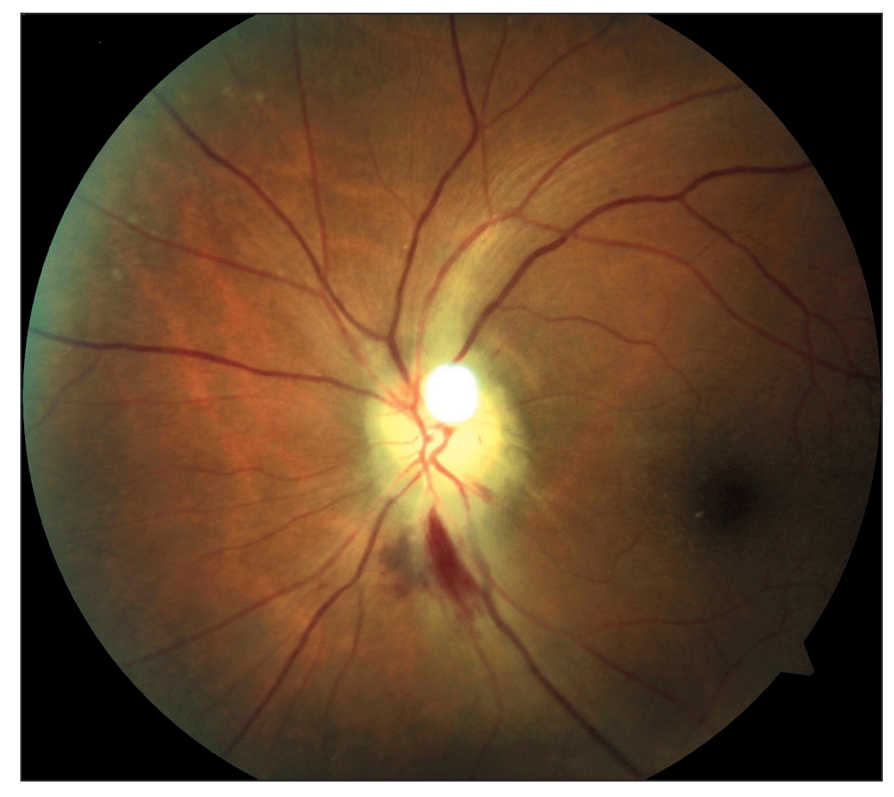

Figura 2. Ojo izquierdo: fondo de ojo enfermo en forma aguda, se observa nervio óptico de coloración blanco amarillenta, con bordes difusos y mal delimitados, hemorragia peripapilar en sector inferior y pérdida de la relación arteria-vena.

\section{Discusión}

La liposucción es el procedimiento estético más común en el mundo para corrección de los defectos del contorno corporal; simplemente al colocar la palabra $l i$ posuction (traducción al ingles del término liposucción) en el analizador de tendencias de Google, encontramos 10.100.000 menciones en menos de 1 minuto, lo que habla de la popularidad del procedimiento en nuestros días.

Todos los cirujanos plásticos que realizan liposucción deben estar familiarizados con los riesgos, secuelas y complicaciones asociadas al procedimiento e informar claramente al paciente. Afortunadamente la gran mayoría de esas complicaciones son menores y se pueden resolver espontáneamente. ${ }^{(4)}$ Hughes demostró que la tasa de complicación aumenta cuando la liposucción es de gran volumen y/o se combina con otros procedimientos quirúrgicos. ${ }^{(5)}$ Las complicaciones mayores, incluyendo perforación de órganos internos, tromboembolismo pulmonar, micro o macro embolia grasa y muerte súbita son más raros, con incidencias reportadas que van del $1 \%$ hasta el $8.5 \% \cdot{ }^{(6,7)}$ En la actualidad se estima que la tasa de mortalidad asociada a un embolismo graso microscópico postliposucción es del 10\% cuando el paciente recibe un tratamiento adecuado. ${ }^{(8)}$

Por su parte, la neuropatía óptica isquémica anterior (NOIA) es un espectro de patologías del nervio óptico reconocidas como la segunda causa más frecuente de patología del disco óptico. En su fisiopatología encontramos 2 variedades: una causada por arteritis secundarias a enfermedades vasculares y otra no arterítica, secundaria a la oclusión de las arterias ciliares posteriores que pro- 
veen la irrigación de la parte anterior de la cabeza del nervio óptico, provocando edema axonal y un síndrome caracterizado por disminución de la agudeza visual total o parcial de manera indolora. ${ }^{(9,10)}$ La neuropatía óptica isquémica anterior (NOIA) perioperatoria como causante de ceguera posterior a liposucción es una eventualidad extremadamente rara, encontrando solamente 7 pacientes reportados en la literatura mundial. ${ }^{(3)}$ Sin embargo, el procedimiento quirúrgico está reconocido como un factor de riesgo para desarrollar embolias grasas. Sabemos de antemano que la NOIA, por las características propias de la cabeza del nervio óptico, es un excelente blanco para desarrollar dicha isquemia secundaria a un émbolo graso.

La NOIA se presenta como una pérdida de la visión no dolorosa a partir de las 48 horas de un postoperatorio. Los cambios visuales pueden ir desde visión sin percepción de la luz y campo visual nulo, hasta varios grados de déficit en el mismo. ${ }^{(3)}$

En la Tabla I, adaptada de la última revisión publicada por Ahmad y Pelak en 2016, sintetizamos las publicaciones previas incluyendo nuestro caso, recogiendo rangos de lipoaspiración que van desde los 2.800 a los
$22.000 \mathrm{ml}$ (media de $3.575 \mathrm{ml}$ ): 5 casos con afectación bilateral y 3 casos, incluyendo el nuestro, de afectación unilateral. De 13 ojos, 2 tuvieron sospecha de neuropatía óptica isquémica posterior (NOIP) y 11 de neuropatía óptica isquémica anterior (NOIA). Seis de los 8 casos presentaron algún grado de anemia o hipotensión que se presentó antes de la perdida de la visión, igual que sucedió en nuestra paciente..$^{(11-16)}$

Los mecanismos específicos que provocan la NOIA perioperatoria son poco claros, pero existen variables que incluyen la posición en decúbito prono durante la cirugía, la pérdida sanguínea, la anemia, hemodilución, hipotensión y el uso de vasopresores. Entre los factores de riesgo del paciente se pueden incluir la diabetes mellitus, la hipertensión arterial o las enfermedades cardiovasculares. $^{(3,9)}$

Creemos que en nuestra paciente, los factores determinantes para la aparición de esta patología fueron secundarios a la presencia de hemodilución, anemia e inflamación generalizada, que aparecen como complicaciones de cualquier liposucción favoreciendo la instalación de un cuadro de embolismo graso con afectación directa de la arteria ciliar anterior.

Tabla I. Revisión bibliográfica de casos

\begin{tabular}{|c|c|c|c|c|c|c|c|c|}
\hline Referencia & Rath et al (11) & Moura et al (12) & $\begin{array}{l}\text { Ribeiro Mon- } \\
\text { teiro et al }{ }^{(13)}\end{array}$ & $\begin{array}{l}\text { Sibgatullah } \\
\text { et al (14) }\end{array}$ & $\begin{array}{l}\text { Foroozan and } \\
\text { Varon }{ }^{(15)}\end{array}$ & Minagar et al (16) & Ahmad et al (3) & $\begin{array}{l}\text { Nuestro } \\
\text { paciente }\end{array}$ \\
\hline $\begin{array}{l}\text { Edad/ sexo } \\
\text { / cirugía }\end{array}$ & $\begin{array}{l}43 \text { años / } \\
\text { F/ Aumento } \\
\text { mamario + } \\
\text { liposucción } \\
\text { abdominal }\end{array}$ & $\begin{array}{l}49 \text { años / F/ } \\
\text { Liposucción } \\
\text { de muslos, } \\
\text { espalda, cade- } \\
\text { ra, resección } \\
\text { de nódulos } \\
\text { mamarios }\end{array}$ & $\begin{array}{l}34 \text { años / F / } \\
\text { Liposucción es- } \\
\text { palda, glúteos + } \\
\text { abdominoplastia }\end{array}$ & $\begin{array}{l}36 \text { años / F / } \\
\text { Liposucción } \\
\text { (Zonas no } \\
\text { reportadas) }\end{array}$ & $\begin{array}{l}30 \text { años / F / } \\
\text { Liposucción de } \\
\text { alto volumen } \\
+ \text { abdomino- } \\
\text { plastia }\end{array}$ & $\begin{array}{l}47 \text { años / F / } \\
\text { Liposucción de } \\
\text { abdomen, muslos } \\
\text { y brazos }\end{array}$ & $\begin{array}{l}38 \text { años / } \\
\text { Liposucción } \\
\text { de espalda, } \\
\text { glúteos, mus- } \\
\text { los + abdomi- } \\
\text { noplastia }\end{array}$ & $\begin{array}{l}32 \text { años / F / } \\
\text { Liposucción de } \\
\text { abdomen y es- } \\
\text { palda + aumento } \\
\text { mamario }\end{array}$ \\
\hline $\begin{array}{l}\text { Volumen } \\
\text { aspirado } \\
(\mathrm{ml})\end{array}$ & 2800 & 3000 & 5500 & $\mathrm{n} / \mathrm{a}$ & 22000 & 3700 & 3450 & 3000 \\
\hline $\begin{array}{l}\text { Factores } \\
\text { de riesgo y } \\
\text { complica- } \\
\text { ciones }\end{array}$ & $\begin{array}{l}\text { Mutación gen } \\
\text { MTHFR (Hiper- } \\
\text { coagulabilidad) }\end{array}$ & $\begin{array}{l}\text { Discos ópticos } \\
\text { pequeños, } \\
\text { gran pérdida } \\
\text { de sangre }\end{array}$ & $\begin{array}{l}\text { Hipertensión } \\
\text { intracraneal } \\
\text { idiopática }\end{array}$ & $\begin{array}{l}\text { Gran pérdida } \\
\text { de sangre, } \\
\text { hemodilución }\end{array}$ & $\begin{array}{l}\text { Hipotiroidismo, } \\
\text { TEP, trombo- } \\
\text { sis de seno } \\
\text { venoso dural, } \\
\text { baja cuenta } \\
\text { plaquetaria, } \\
\text { anemia }\end{array}$ & $\begin{array}{l}\text { Gran pérdida } \\
\text { sanguínea, ane- } \\
\text { mia, taquicardia, } \\
\text { hipotensión }\end{array}$ & $\begin{array}{l}\text { Anemia, } \\
\text { hipotensión, } \\
\text { posición prono } \\
\text { transopera- } \\
\text { toria }\end{array}$ & $\begin{array}{l}\text { Posición prono } \\
\text { transoperatoria } \\
\text { anemia postoper- } \\
\text { atoria }\end{array}$ \\
\hline Diagnóstico & NOIP derecha & NOIA bilateral & NOIA bilateral & $\begin{array}{l}\text { NOIA izqui- } \\
\text { erda, prob- } \\
\text { able NOIP } \\
\text { derecha }\end{array}$ & NOIA bilateral & NOIA derecha & NOIA bilateral & NOIA izquierda \\
\hline Tratamiento & Prednisona IV & $\begin{array}{l}\text { Prednisona IV } \\
\text { y oral }\end{array}$ & $\begin{array}{l}\text { Acetazolamida } \\
\text { oral }\end{array}$ & $\begin{array}{l}\text { Transfusión } \\
\text { sanguínea } \\
17 \text { días PO }\end{array}$ & $\begin{array}{l}\text { Acetazolamida } \\
\text { oral + trans- } \\
\text { fusión san- } \\
\text { guínea }\end{array}$ & $\begin{array}{l}\text { Prednisona oral + } \\
\text { transfusión san- } \\
\text { guínea }\end{array}$ & $\begin{array}{l}\text { Transfusión } \\
\text { sanguínea + } \\
\text { brimonidine } \\
\text { tópico }\end{array}$ & $\begin{array}{l}\text { Transfusión } \\
\text { sanguínea + } \\
\text { prednisona oral }\end{array}$ \\
\hline $\begin{array}{l}\text { Resultado } \\
\text { visual }\end{array}$ & $\begin{array}{l}20 / 80 \text { ojo dere- } \\
\text { cho con esco- } \\
\text { toma central }\end{array}$ & $\begin{array}{l}20 / 25 \text { ambos } \\
\text { ojos, defecto } \\
\text { bilateral inferior } \\
\text { altitudinal }\end{array}$ & $\begin{array}{l}\text { Cuenta dedos } \\
\text { ojo derecho, } \\
20 / 200 \text { ojo } \\
\text { izquierdo }\end{array}$ & $\begin{array}{l}20 / 20 \text { con } \\
\text { constricción } \\
\text { periférica de } \\
\text { campo visual } \\
\text { ojo derecho, } \\
20 / 25 \text { con } \\
\text { defecto } \\
\text { altitudinal } \\
\text { inferior ojo } \\
\text { izquierdo }\end{array}$ & $\begin{array}{l}20 / 50 \text { ojo } \\
\text { derecho, } 20 / 60 \\
\text { ojo izquierdo, } \\
\text { defecto altitu- } \\
\text { dinal inferior y } \\
\text { defecto arcuato } \\
\text { superior en } \\
\text { ambos ojos }\end{array}$ & $\begin{array}{l}\text { Sin percepción } \\
\text { de luz ojo dere- } \\
\text { cho }\end{array}$ & $\begin{array}{l}20 / 20 \text { con leve } \\
\text { constricción de } \\
\text { campo visual } \\
\text { periférico ojo } \\
\text { derecho, } 20 / 25 \\
\text { con defecto } \\
\text { altitudinal } \\
\text { inferior ojo } \\
\text { izquierdo }\end{array}$ & $\begin{array}{l}20 / 20 \text {, recu- } \\
\text { peración sin } \\
\text { secuelas apar- } \\
\text { entes }\end{array}$ \\
\hline
\end{tabular}


Desafortunadamente, no existe un tratamiento consensuado o establecido que altere el curso de la NOIA y solamente la prevención es la clave para disminuir la morbilidad relacionada a la cirugía. La corrección de la hipotensión y la anemia, aunque necesarias, tienen una influencia poco clara en el pronóstico visual cuando la ceguera ha ocurrido. ${ }^{(17)}$

Los tratamientos posteriores a la perdida de la visión incluyen esteroides sistémicos, acetazolamida y transfusiones sanguíneas. El uso de esteroides sistémicos sigue siendo un arma para contrarrestar la patología óptica, ${ }^{(18)}$ sin embargo, a pesar de los tratamientos iniciados después de la aparición de los síntomas, en algunos casos no se ve una influencia favorable en el resultado visual ${ }^{(3)}$ ya que se ha demostrado que solo hay mejoría de campos visuales y agudeza visual en pacientes con agudezas peores de 20/70. En nuestra paciente, la recuperación de la visión fue absoluta (20/20) después de 2 meses de tratamiento, reponiendo de manera inicial la formula roja y estableciendo un tratamiento agresivo con esteroides sistémicos durante 7 semanas para lograr una correcta evolución del padecimiento. Así, observamos de acuerdo a la la literatura que a las 4 a 8 semanas se produce la recuperación de la agudeza visual de forma paulatina, junto con la recuperación de las características normales del nervio óptico. Es por ello que se deben realizar estudios funcionales, como el campo visual, para conocer de manera objetiva tanto el diagnóstico del paciente como su recuperación al implementar el tratamiento sistémico, sin olvidar las pruebas para discriminación de colores y de agudeza visual.

Para la prevención de complicaciones debemos seguir las recomendaciones para liposucción del Comité de Seguridad de la Asociación Mexicana de Cirugía Plástica, Estética y Reconstructiva, entre las que destacan la selección adecuada del paciente, la realización de valoración preanestésica y cardiovascular, no sobrepasar los 3 litros de lipoaspiración con técnica tumescente, mantener al paciente en quirófano sin cambios de temperatura, controlar un adecuado balance de líquidos, utilizar medias de compresión y compresores neumáticos, aplicar hemoconcentrados en los casos necesarios, así como seguir las guías clínicas para el uso de anticoagulación cuando el paciente lo amerite.

\section{Conclusiones}

Mostramos nuestra experiencia con el primer caso reportado en México y octavo en el mundo de ceguera legal unilateral ocurrido después de una liposucción en una mujer de mediana edad y sin antecedentes de importancia.
La paciente evolucionó favorablemente después de la detección temprana de la patología y del tratamiento agresivo con esteroides sistémicos, recuperando la visión y las características normales del nervio óptico a los 2 meses de la intervención.

Se trata de una complicación muy rara pero latente, por lo que proponemos que tal vez se debería tener en cuenta en el consentimiento informado.

\section{Dirección del autor}

Dr. Raúl Alfonso Vallarta Rodríguez

Puente de Piedra 150-419 T2

Tlalpan C.P: 14050

Ciudad de México,

Correo electrónico: avallarta1@gmail.com

\section{Bibliografía}

1.- American Society for Aesthetic Plastic Surgery. 2016 Cosmetic Surgery National Data Bank Statistics: https://www.surgery. org/sites/default/files/ASAPS-Stats2016.pdf

2.- Bellini E, Grieco M, Raposio E. A journey through liposuction and liposculture: Review. Ann Med Surg (Lond). 2017; 24: 5360.

3.- Ahmad M, Pelak V. Vision Loss in a Young, Healthy Woman following liposuction. Am J of Cosmetic Surg. 2016; 33(2): 96101.

4.- Matarasso A, Levine SM. Evidence-based medicine: liposuction. Plast Reconstr Surg. 2013;132(6): 1697-1705.

5.- Hughes CE. Reduction of lipoplasty risks and mortality: An ASAPS survey. Aesthet Surg J. 2001;21(2):120-127.

6.- Miszkiewicz K, Perreault I, Landes G, et al. Venous thromboembolism in plastic surgery: Incidence, current practice and recommendations. J Plast Reconstr Aesthet Surg. 2009;62:580588.

7.- Durán H, Cárdenas-Camarena L, Bayter-Marin JE, Ramos-Gallardo G, Robles-Cervantes JA. Microscopic and Macroscopic Fat Embolism: Solving the Puzzle with Case Reports. Plast Reconstr Surg. 2018; 142(4):569-577.

8.- De Jong RH, Grazer FM. Perioperative management of cosmetic liposuction. Plast Reconstr Surg. 2001;107(4):1039-1044.

9. Miller NR, Arnold AC. Current concepts in the diagnosis, pathogenesis and management of non-arteritic anterior ischemic optic neuropathy. Eye (Lond). 2015;29(1): 65-79.

10. Berry S, Lin WV, Sadaka A, Lee AG. Non-arteritic anterior ischemic optic neuropathy: cause, effect, and management. Eye Brain. 2017; 9: 23-28.

11.- Rath EZ, Falick Y, Rumelt S. Posterior ischemic optic neuropathy following breast augmentation and abdominal liposuction. Can J Ophthalmol. 2009;44: 346-347.

12.- Moura FC, Cunha LP, Monteiro ML. Bilateral visual loss after liposuction: case report and review of the literature. Clinics 2006;61: 489-491

13.- Ribeiro Monteiro ML, Moura FC, Cunha LP. Bilateral visual loss complicating liposuction in a patient with idiopathic intracranial hypertension. J Neuroophthalmol. 2006;26: 34-37.

14.- Sibgatullah M, Kupersmith MJ, Zerykier A, Volpe S. Ischemic optic neuropathy after liposuction: case report and review. Neuro Ophthalmol. 2005;29: 91-93.

15.- Foroozan R, Varon J. Bilateral anterior ischemic optic neuropathy after liposuction. J Neuroophthalmol. 2004;24: 211-213. 
16.- Minagar A, Schatz NJ, Glaser JS. Liposuction and ischemic optic neuropathy: case report and review of literature. $J$ Neurol Sci. 2000;181: 132-136.

17.- Roth S. Perioperative visual loss: what do we know, what can we do?. Br J Anaesth. 2009;103(1): 31-40.
18.- Hayreh SS, Zimmerman MB. Non-arteritic anterior ischemic optic neuropathy: role of systemic corticosteroid therapy. Graefes Arch Clin Exp Ophthalmol. 2008;246(7): 1029-1046. 